\title{
Über eine besondere Lagenbeziehung von zwei Oberflächen zweiter Ordnung.
}

\author{
Von Gustav Kohn in Wien.
}

Die besondere Lagenbeziehung von zwei Kegelschnitten, welche durch die Existenz von unendlich vielen, dem einen Kegelschnitt eingeschriebenen und zugleich dem anderm umschriebenen Dreiecken charakterisiert ist, gehört zu den bekanntesten Beziehungen, welche zwei Kegelschnitte gegen einander aufweisen können. Es liegt nahe, nach räumlichen Analogien zu suchen, und Hurwitz hat bekanntlich ${ }^{1}$ ) eine solche in der nach ihm benannten Beziehung von zwei cubischen Raumcurven gefunden, welche durch die Existenz unendlich vieler der einen eingeschriebener und zugleich der anderen umschriebener Tetraeder definiert ist.

Man kann aber die Frage aufwerfen, ob die Figur der beiden Kegelschnitte der Ebene nicht auch ein räumliches Analogon besitzt, das an die Stelle der Kegelschnitte Oberflächen zweiter Ordnung treten lässt.

Die Beantwortung dieser Frage kann man zunächst in der Richtung suchen, welche sich direct darbietet. Da es für zwei beliebige Oberflächen zweiter Ordnung stets und zwar $\infty^{ \pm}$Tetraeder gibt, welche der einen eingeschrieben und zugleich der anderen umschrieben sind, so wird man nach der Lagenbeziehung der zwei Flächen fragen, für welche $\infty^{5}$ solche Tetraeder existieren. Diese Frage hat erst kürzlich Hr. G. Fonten é aufgeworfen und dahin beantwortet, dass die beiden Oberflächen zweiter Ordnung Vierseitsdurchschnitt aufweisen müssen. ${ }^{2}$ )

Man kann aber auch einen anderen indirecten Weg einschlagen. Man kann statt der ursprünglich zur Definition der Lagenbeziehung der beiden Kegelschnitte zugrunde gelegten Eigenschaft eine andere diese Beziehung ebenfalls definierende Eigenschaft bei der Ü̉bertragung in den Raum zum Ausgangspunkt nehmen.

Zwei Kegelschnitte in der betrachteten Lagenbeziehung kann man durch eine eigenthümliche projective Erzeugung definieren,

1) Math. Annalen Bd. 20.

2) Siehe Nouvelles Annales, Februar 1899. 
welche sie zulassen. Durch Annahme einer projectiven Beziehung: zwischen zwei Strahlenbüscheln $S$ und $S^{\prime}$. der Ebene sind auch irgend zwei Punktreihen $u$ und $u$ der Ebene projectiv auf einander bezogen, sobald man die Gerade $u$ mit dem Strahlenbüschel $S$, die Gerade $u^{\prime}$ mit dem Strahlenbüschel $S^{\prime}$ zum Durchschnitt bringt und die Schnittpunkte entsprechender Strahlen einander als entsprechend zuweist. Die beiden Kegelschnitte, von denen der eine als Erzeugnis der beiden projectiven Strahlenbüschel $S$ und $S^{\prime}$, der andere als Erzeugnis der beiden projectiven Punktreihen $u$ und $u^{\prime}$ hervorgeht, stehen in der Lagenbeziehung; welche wir betrachten; umgekehrt lassen aber zwei Kegelschnitte in dieser Lagenbeziehung stets und zwar noch auf $\infty^{2}$ Arten die in Rede stehende Erzeugung zu. Als Scheitel $S, S^{\prime}$ der den einen Kegelschnitt erzeugenden projectiven Strahlenbüschel hat man zwei Ecken von zwei ihm ein- und dem andern Kegelschnitt umschriebenen Dreiecken zu wählen, als die Trïger $u, u^{\prime}$ der den andern Kegelschnitt erzeugenden projectiven Punktreihen ihre Gegenseiten in diesen Dreiecken und zwar. $u^{\prime}$ als Gegenseite von $S$ and $u$ als Gegenseite von $S^{\prime}$.

Definiert man die Lagenbeziehung der beiden Kegelschnitte durch diese Erzeugung, so bieten sich für die Übertragung in den Raum sofort zwei Wege dar. Der eine lässt uns die beiden projectiven Strahlenbüschel durch zwei collineare Strahlenbündel, die beiden Punktreihen durch ebene Felder ersetzen und führt zu zwei eubischen Raumcurven in der $\mathrm{Hurwitz}$ 'schen Lagenbeziehung. Man erkennt auch, dass irgend zwei solche cubische Raumcurven auf $\infty^{2}$ Arten in der gedachten Weise erzeugt werden können. Als Scheitel der die eine Curve erzeugenden collinearen Strahlenbündel hat man zwei Ecken von zwei dieser Curve eingeschriebenen und der anderen umschriebenen Tetraedern anzunehmen, als Träger der die zweite Curve erzeugenden collinearen Felder aber die Gegenebenen dieser Eckpnnkte in den beiden Tetraedern und zwar als erste Ebene die Gegenebene des zweiten Eckpunktes1).

Man kann aber auch - und dies soll im Folgenden unternommen werden - an die Stelle der zwei projectiven Strahlenbüschel. der Ebene im Raume zwei projective Ebenenbüschel setzen und statt der Punktreihen der Ebene im Raume ebenfalls Punktreihen wählen.

Wir haben dann zwei projective Ebenenbüschel, ihre Axen sollen $v$ und $v^{\prime}$ heißen, welche eine Fläche zweiter Ordnung erzeugen, die mit $\Phi$ bezeichnet werden soll, und außerdem zwei Punktreihen $u$ und $u^{\prime}$, welche dadurch projectiv anf einander bezogen sind, dass dem Schnittpunkte einer beliebigen Ebene des Büschels $v$ mit der Geraden $u$ der Schnittpunkt der ihr im Büschel $v^{\prime}$ entsprechenden Ebene mit der Geraden $u^{\prime}$ zugewiesen ist. Die

1) Für diese und die vorstehende Bemerkung mag der Nachweis seiner Einfachheit halber unterdrückt werden. 
Fläche zweiter Ordnung, welche als Frzeugnis der projectiven Punktreihen $u$ und $u^{\prime}$ auftritt, nennen wir $F$.

Die erste Frage gilt nun einer geometrischen Beziehung der Flächen $\Phi$ und $F$.

Unter den Verbindungslinien entsprechender Punkte von $u$ und $u^{\prime}$ wird es zwei geben, sie sollen $a_{1}$ und $a_{2}$ heißen, welche die Gerade $v$ und zwei, sie sollen $a_{1}^{\prime}, a_{2}^{\prime}$ heißen, welche die Gerade $v^{\prime}$ treffen. Man kann nun leicht zeigen, dass das windschiefe Viereck, ron dem $v$ und $u^{\prime}$ das eine, $a_{1} a_{2}$ das andere Gegenkantenpaar darstellen, Eckpunkte besitzt, die den beiden Flächen $\Phi$ und $F$ angehören, aud dass ein Gleiches rom Vierecke gilt, das die Strahlenpaare $v^{\prime}$, $u$ und $a_{1}^{\prime}, a_{2}^{\prime}$ zu Gegenkantenpaaren hat.

Um dies z. B. für das erste Viereck einzusehen, beachte man, dass die beiden auf der Geraden $v$ gelegenen Ecken dieses Vierecks den beiden Flächen $\Phi$ und $F$ angehören, weil sie als Schnittpunkte der Geraden $v$ der Fläche $\Phi$ mit den Geraden $a_{1}, a_{2}$ der Fläche $E$ auftreten. Die beiden Punkte $u^{\prime} a_{1}$ und $u^{\prime} a_{2}$ liegen als in der Geraden $a_{1}$ beziehungsweise $a_{2}$ enthalten auf der Fläche $F$. Auf der Fläche $\Phi$ wird aber jeder ảieser beiden Punkte liegen, weil die beiden Ebenen, welche ihn mit den Geraden $v$ und $v^{\prime}$ verbinden, entsprechende Ebenen in den beiden projectiven Ebenenbüscheln $v$ und $v^{\prime}$ sein werden, als deren Erzeugnis die Fläche $\Phi$ definiert wurde. Denn die Gerade $a_{1}$ (und ebenso $a_{2}$ ) verbindet zwei entsprechende Punkte der projectiven Punktreiben $u$ und $u^{\prime}$ und es werden diese Pankte aus $v$ und $v^{\prime}$ projiciert entsprechende Ebenen der beiden Ebenenbiischel ergeben.

Wir haben jetzt die Existenz von zwei windschiefen, Vierecken erschlossen, welche den Flächen $\Phi$ und $F$ zu gleicher Zeit eingeschrieben sind, und von deren Kanten eine der einen, die drei übrigen der anderen Fläche angehören. Es lässt sich nun zeigen, dass die Existenz eines solchen Vierecks die Existenz unendlich vieler solcher Vierecke nach sich zieht und die Lagenbeziehung der zwei. Flächen zweiter Ordnung vollstïndig charakterisiert.

Um den Nachweis für diese Behataptung elementar zu führen, gehen wir von einem beliebigen windschiefen Viereck aus und bezeichnen, um mit den obigen Bezeichnungen in Übereinstimmung zu bleiben, zwei Gegenkanten des Vierecks mit $v$ und $u$, die beiden anderen mit $a_{1}$ und $a_{2}$. $\Phi$ soll eine beliebige dom Viereck umschriebene Fläche zweiter Ordnung bedeuten, welche durch die Gerade $v$ hindurchgeht und $F$ eine beliebige Fläche zweiter Ordnung, welche die drei übrigen Kanten des Vierecks enthält. Mit $v^{\prime}$ wollen wir jetzt eine beliebigo Erzeugende der Fläche $\Phi$ bezeichnen, welche derselben Schaar angehört, wie die Erzeugende $v$ dieser Fläche. Dann können wir, wie gezeigt werden soll, ein windschiefes Viereck construieren, dass zu den Flächen $\phi$ und $F$ dieselbe Beziehung hat wie das angenommene und die Gerade $v^{\prime}$ als eine Kante an Stelle der Geraden $v$ enthält. 
Die Gerade $v^{\prime}$ wird von zwei Erzeugenden der Fläche $F$, sie. mögen $a_{1}^{\prime}, a_{2}^{\prime}$ heißen, getroffen werden, welche derselben Schaar wie die Erzengenden $a_{1}, a_{2}$ angehören. Eine von jenen zwei Erzeugenden, z. B. $a_{1}^{\prime}$, wird die Fläche $\Phi$ auber in einem Punkte der Geraden $v^{\prime}$ noch in einem zweiten Punkte treffen und durch diesen wird neben $a_{1}^{\prime}$ noch eine zweite Erzeugende der Fläche $F$ hindurchgehen, die wir mit $u$ bezeichnen.

Denkt man sich nun die Fläche $\Phi$ erzeugt durch zwei projective Ebenenbüschel, deren Axen die Erzeugenden $v$ und $v^{\prime}$ derselben Schaar sind, so kann man eine projective Beziehung zwischen den Punktreihen $u$ und $u^{\prime}$ dadurch herstellen, dass man die erste mit dem Ebenenbüschel $v$, die zweite mit dem Ebenenbüschel $v^{\prime}$ zum Durchschnitt bringt und die Schnittpunkte entsprechender Ebenen einander als entsprechend zuweist. Das Erzeugnis der in dieser Weise projectiv auf einander bezogenen Punktreihen $u$ und $u^{\prime}$ kann nun nicht verschieden sein von der Fläche $F$, weil unsere Annahmen so getroffen sind, dass die drei Erzeugenden von $F: a_{1}, a_{2}, a_{1}^{\prime}$ entsprechende Punkte der beiden projectiven Punktreihen verbinden. Wenn aber die Flächen $\Phi$ und $F$, wie es jetzt deutlich ist, die Erzeugung zulassen, von der wir oben ausgegangen sind, so gilt das oben aus dieser Erzeugung abgeleitete Ergebnis, dass nicht nur die vier Geraden $v a_{1} u^{\prime} a_{2}$, sondern auch die vier Geraden $v^{\prime} a_{1}^{\prime} u a_{2}^{\prime}$ ein windschiefes Viereck darstellen, dessen Ecken auf beiden Flächen liegen und von dessen Kanten die eine $v^{\prime}$ der Fläche $\Phi$, die andẻren der Fläche $F$ angehören.

Da. $v^{r}$ eine beliebige Erzeugende der einen Schaar von $\Phi$ war, so ist die Existenz von $\infty^{1}$ windschiefen Vierecken der eben angegebenen Beschaffenheit nachgewiesen. Da man $v a_{1} u^{\prime} a_{2}$ und $v^{\prime} a_{1}^{\prime}$ $u a_{2}^{\prime}$ als zwei beliebige unter diesen windschiefen Vierecken ansehen kann, so ergibt sich ferner, dass je zwei von diesen $\infty^{1}$ Vierecken zu einer projectiven Erzeugung der Flächen $\Phi$ und $F$ führen, von der besonderen Art, wie sie oben betrachtet wurde.

Die Eigenthümlichkeit dieser Erzeugungsart besteht darin, dass die zwei projectiven Ebenenbüschel, welche die eine Fläche erzeugen, zu den die zweite Fläche erzeugenden zwei projectiven Punktreihen in der Beziehung stehen, das zwei incidenten Elementen des ersten Ebenenbüschels und der ersten Punktreihe im zweiten Ebenenbüschel und der zweiten Punktreihe immer wieder zwei ineidente Elemente entsprechen. Daraus erhellt, dass diese Erzeugungsart für ein Flächenpaar zweiter Ordnung zu sich selbst dualistisch ist, dass aber durch die dualistische Erzeugung die beiden Flächen in verkehrter Reihenfolge hervorgehen. Infolge dessen steht jeder Eigenschaft eines so erzeugten Flächenpaares zweiter Ordnung eine zweite. nach dem Dualitätsgesetz unter Vertauschung der beiden Flächen aus ihr hervorgehende gegenüber, eine Bemerkung, der wir weiter unten bei der Formulieruug der erlangten Ergebnisse Rechnung tragen. 
Vorher schalten wir den Nachweis durch Constantenzählung dafür ein, dass die Möglichkeit unserer Erzeugung für ein Flächenpaar zweiter Ordnung eine einfache Bedingung involviert. Die Erzeugang hängt von 19 Constanten ab, da jede der vier Geraden, von denen zwei als die Axen projectiver Ebenenbüschel, zwei als die Träger projectiver Punktreihen auftreten, vier Constanten anbaften und die Feststellung der projectiven Beziehung der Ebenenbüschel drei weitere mit sich bringt. Von diesen 19 Constanten sind zwei in Abzug zu bringen, weil bewiesen wurde, dass ein Flächenpaar, das die gedachte Erzeugung zulässt, dies stets auf $\infty^{2}$ Arten thut, so dass unser Flächenpaar von 17 Constanten abhängt, also von einer weniger als ein beliebiges Paar von Oberflächen zweiter Ordnung.

Unser Ergebnis lautet:

Es ist eine einfache Bedingung für zwei Oberflächen zweiter Ordnung, wenn es entweder ein beiden Flächen eingeschriebenes einfaches Viereck geben soll, von dessen Kanten eine der ersten, die drei übrigen der zweiten Fläche angehören, oder ein beiden Flächen umschriebenes einfaches Vierflach von dessen Kanten drei der ersten und eine der zweiten angehört. Es gibt dann immer so wohl $\infty^{1}$ solche Vierecke als auch $\infty^{1}$ solche Vierflache.

In jedem dieser Vierecke (Vierflache) gibt es zwei Gegenkanten, von denen jede einer anderen von den zwei Flächen angehört und zwei Paare von solchen zwei Gegenkanten stellen immer die Träger der projectiven Grundgebilde einer solchen besonderen Erzeugung der beiden Flächen dar, durch die wir deren Lagenbeziehung ursprünglich definiert haben. 